\title{
Etude Archeothanatologique De La Sepulture Protohistorique Du Monument Funeraire Mbii (Boulmane, Moyen Atlas, Maroc)
}

\section{Benlamine Lalla khaddouje, Dr.} Benabdelhadi Mohammed, Pr.

Laboratoire des Géo-Ressources et Environnement. Université Sidi Mohammed Ben Abdellah, Fès, Maroc

Oujaa Aicha, Pr.

Institut Nationale des Sciences de l'Archéologie et du Patrimoine, Rabat, Maroc

Azzouzi Nassareddine, Pa.

Laboratoire Ressources Naturelles et Environnement,

Faculté polydisciplinaire, Taza, Maroc

\section{Benabdelhadi Soumaya}

Rotterdam School Of Management, Erasmus Universiteit,

Rotterdam, Netherlands

Fontugne Michel, Pr.

Laboratoire des Sciences du Climat et de l'Environnement,

Gif / Yvette, France

doi: 10.19044/esj.2017.v13n11p146 URL:http://dx.doi.org/10.19044/esj.2017.v13n11p146

\begin{abstract}
The Moudmane Boulama site is a funeral structure located at $10 \mathrm{~km}$ South West of the city of Boulmane (Middle Atlas). It is a necropolis made of approximately twenty tumuli implanted on a hill. The MBII funeral monument is a tumulus built on the top of this hill, which culminates at 1970 $\mathrm{m}$. It is an oval formation made of stack of stones and blocks. The funeral space is delimited in its West part by a low wall made of a sharpened stone alignment. The digs undertaken in June 2013 brought to light the burial place of a $\mathrm{H} 3$ adult and some remains of another adult as well as an immature subject.

The skeleton of the most complete $\mathrm{H} 3$ individual is a male adult, buried in a lying dorsal position, oriented towards East West, head towards West and feet East. He is in a hyper contracted position and lays on an oval plane pit with a diameter of less than a meter. The calvarium and the mandible were subjected to a rotation and lay on the right side. The superior members are
\end{abstract}


folded on themselves and laid on the thorax. The inferior members are also flexed. The skeleton is characterised by the maintaining of the connection of a number of labile elements which allows the characterisation of the burial place as being primarily in clogged space. This funeral monument has probably been used at first for the reception of the two individuals (H1 and $\mathrm{H} 2$ ) and then for the burial of the H3 individual in a sepulchral pit. The reuse of the monument could be explained by the three individuals belonging to the same family and that the last buried subject H3 has a more important familial and possibly social role. This tumulus has also released funeral furniture made of a few bones of domestic fauna. They were the object of a radiometric dating, which allowed the attribution of $2290 \pm 35$ years old to this tumulus ${ }^{14}$ C B.P (GifA15085/ Sac A 41509).

Keywords : Moudmame Boulama, necropolis, funeral rite, burial place, Middle Atlas

\section{Résumé}

Le site de Moudmane Boulama est une structure funéraire située à une dizaine de kilomètres au sud-ouest de la ville de Boulmane (Moyen Atlas). C’est une nécropole constituée d'une vingtaine de tumuli implantés sur une colline. Le monument funéraire MBII est un tumulus construit sur le sommet de cette colline qui culmine à $1970 \mathrm{~m}$. Il s'agit d'une formation ovalaire faite d'entassement de pierres et de blocs. L'espace funéraire est délimité dans sa partie ouest par un muret constitué d'un alignement de pierres taillées. Les fouilles entreprises en juin 2013 ont mis au jour la sépulture d'un adulte H3 et quelques restes d'un autre adulte, et d'un sujet immature.

Le squelette de l'individu le plus complet $\mathrm{H} 3$ est un adulte de sexe masculin, inhumé en décubitus dorsal, orienté est-ouest, la tête à l'ouest et les pieds à l'est. Il est en position hyper contractée et repose sur une fosse à plan ovalaire d'un diamètre ne dépassant pas un mètre. Le calvarium et la mandibule ont subi une rotation et reposent sur le côté droit. Les membres supérieurs sont repliés sur eux mêmes et déposés sur le thorax. Les membres inférieurs sont également fléchis. Le squelette se caractérise par le maintien en connexion d'un certain nombre d'éléments labiles ce qui permet de définir la sépulture comme étant primaire en espace colmaté. Ce monument funéraire à probablement servi dans un premier temps pour l'accueil des deux individus (H1 et H2) et ensuite pour l'inhumation de l individu H3 dans une fosse sépulcrale. La réutilisation du monument pourrait s'expliquer par l'appartenance des trois individus à la même famille, et que le dernier sujet inhumé $\mathrm{H} 3$ occuperait une position familiale et probablement sociale plus importante. Ce tumulus a également livré un mobilier funéraire constitué de 
quelques ossements de faune domestique. Ils ont fait l'objet d'une datation radiométrique qui permet d'attribuer à ce tumulus l'âge de $2290 \pm 35$ ans ${ }^{14} \mathrm{C}$ B.P (Gif A15085/ Sac A 41509).

Mots clés : Moudmame Boulama, nécropole, rite funéraire, sépulture, Moyen Atlas

\section{Introduction}

Dans le cadre d'un programme de recherche national qui vise la connaissance et la valorisation du patrimoine archéologique du Moyen Atlas. Plusieurs nécropoles ont été découvertes dans la région de Boulmane (Benabdelhadi et al, 2008). Comme la plupart des tumuli, le monument funéraire Moudmame Boulama II se présente perché au sommet d'une colline et correspond à une accumulation de pierres calcaires et gréseuses de dimensions différentes. Il se distingue par la présence d'un muret de soutènement et par des dimensions relativement plus importantes.

En juin 2013, nous avons programmé une compagne de fouille, en adoptant la méthode de l'anthropologie de terrain qui vise à restituer l'attitude originelle du corps, l'agencement des pièces d'habillement, les éléments de parure et du mobilier ce qui contribue aussi à définir l'architecture du tumulus en précisant le milieu de décomposition (espace vide ou colmaté) et en montrant les effets du contenant sur la position des ossements (Duday et al, 1990). Le démontage progressif du tumulus nous a permis de découvrir trois niveaux ; le dernier niveau (niveau III) est constitué de dalles calcaires de forme rectangulaire. Il a mis au jour des vestiges de deux individus incomplets; un adulte (H1) et un immature (H2). Les ossements recueillis ne présentent aucune connexion anatomique et ont été déposés dans des endroits différents du tumulus.

La fosse sépulcrale abrite une sépulture individuelle primaire dans un espace colmaté d'un sujet adulte (H3) de sexe masculin inhumé en décubitus dorsal hyper contracté. Le squelette se présente en positon de flexion forcée due à l'étroitesse de la fosse, qui a une forme ovalaire et présente un fond oblique et peu profond. Le squelette est complet et se caractérise par le maintien d'un certain nombre d'éléments labiles. Nous sommes donc en présence d'une sépulture individuelle primaire dans un espace colmaté (Duday et al, 1990). Les ossements sont généralement en bon état de conservation, seuls la scapula gauche et quelques vertèbres présentent un état de fracturation avancée.

Le monument funéraire a également livré un mobilier funéraire constitué d'un bois, des dents isolés et une partie du crâne d'une mégafaune domestique de type Bos. La datation radiométrique des ossements fauniques 
fait remonter le tumulus à l'âge de $2290 \pm 35$ ans ${ }^{14}$ C B.P (Gif A15085/ Sac A 41509).

\section{Situation géographique}

Le site Moudmame Boulama appartient à la commune rurale d'Essaf dans le Moyen Atlas. Il se situe à une dizaine de kilomètre au sud -ouest de la ville de Boulmane (fig.1). C’est une colline nécropole orientée nord-sud qui s'étend sur plus de $1500 \mathrm{~m}$ de long et présente vingt tumuli implantés sur les lignes de crêtes et sur les flancs de versants (Benabdelhadi et al, 2008).

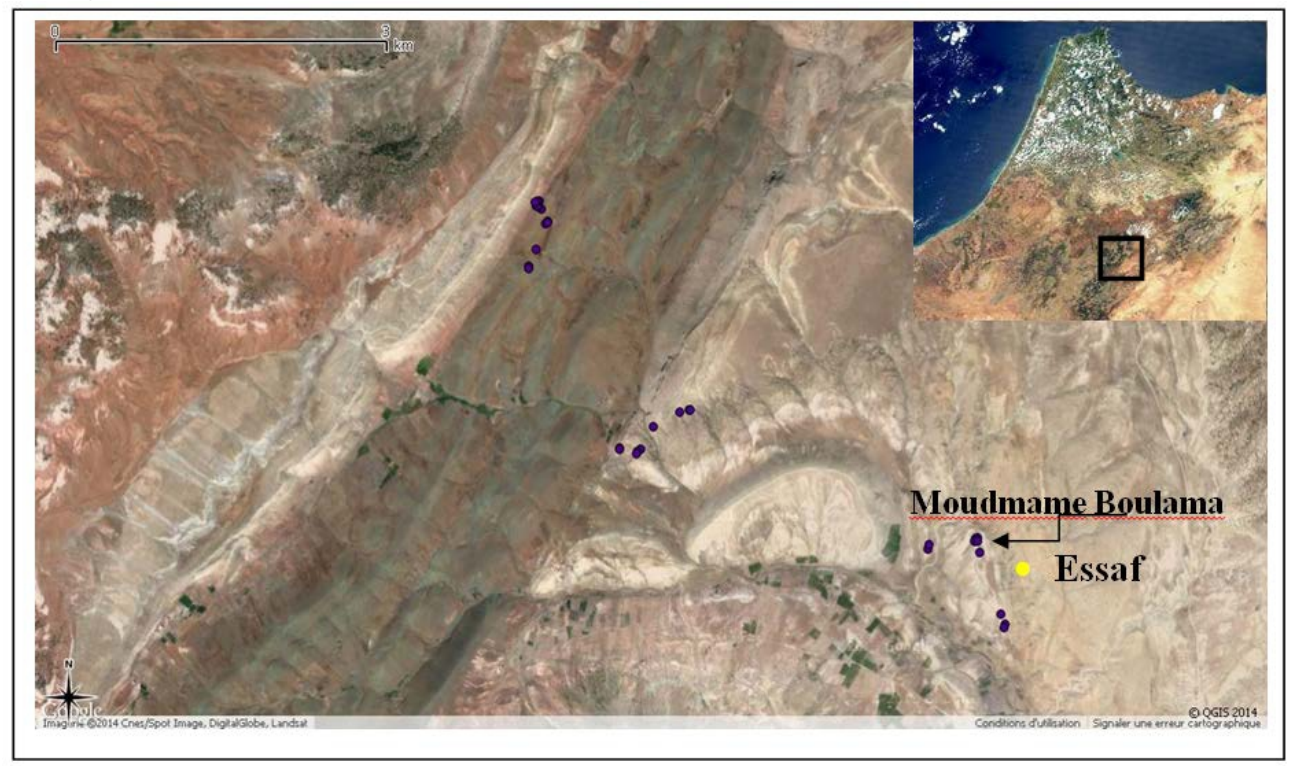

Figure.1. Localisation de la nécropole de Moudmame Boulama, chaque point correspond à une nécropole.

\section{Nécropole Moudmame Boulama}

La nécropole de Moudmame Boulama se présente comme la majorité des tombes à tradition préislamiques par des tumuli. Ces derniers correspondent à des amas de pierres de dimensions et de nature différentes. C’est un ensemble de tumuli de forme circulaire, implantés les uns à côté des autres sur les lignes de crêtes et aux sommets des collines. Leurs hauteurs varient d'un à trois mètres et leurs diamètres sont généralement compris entre six et huit mètres, mais ils peuvent atteindre parfois quinze mètres. Ce sont généralement des espaces protégés qui offrent une conservation optimale pour les ossements humains et pour le mobilier funéraire. Certains tumuli ont été soit pillés par les chercheurs de trésors, soit démantelés par les indigènes pour récupérer les matériaux (Benabdelhadi et al, 2008).

\section{Moudmame Boulama I}

Le tumulus Moudmame Boulama I a été découvert dans la région en 2007. La fouille de sauvetage a mis au jour des restes humains de deux 
individus orientés NW-SE (fig. 4). L'individu adulte de sexe masculin présente une inhumation primaire dans une fosse avec la tête orientée vers le NW. Le maintien des scapulas en position oblique montre que la décomposition a été effectuée dans un milieu colmaté (Benabdelhadi et $a l$, 2007). Le deuxième individu est un adolescent de sexe féminin, inhumé en décubitus latéral droit. Cette adolescente porte des objets de parures en cuivre et en cornaline.
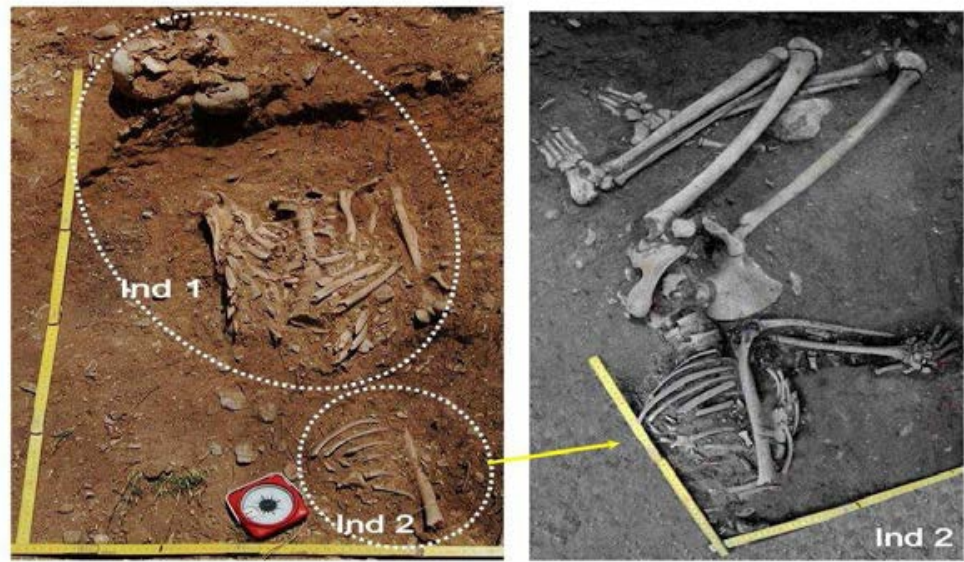

Figure 4. Sépulture de MBI : individu 1(masculin), individu 2 (féminin), (Cliché Benabdelhadi M.et Oujaa A.)

\section{Monument funéraire Moudmame Boulama II Méthode de fouille}

La fouille a été menée par le système de quarts. Nous avons divisé le tumulus en quatre parties suivant les axes nord-sud et est-ouest. Dans un premier temps nous avons commencé par l'enlèvement des pierres qui constituent la partie centrale du quart sud-ouest. Puis nous avons effectué des décapages successifs avec enregistrements graphiques et photographiques. Nous avons procédé à un démontage accompagné d'une identification sur place des ossements. Cette méthode nous a contraints à démonter la structure du tumulus pour accéder aux niveaux plus profonds.

\section{Architecture du tumulus Forme}

Le monument funéraire Moudmame Boulama II se trouve au sommet d'une colline qui culmine à 1970m. C'est une structure ovalaire faite d'une accumulation de pierres et de blocs, d'une longueur de $11,90 \mathrm{~m}$, une largeur de $10,40 \mathrm{~m}$ et une hauteur de $1,70 \mathrm{~m}$. L'érosion de la partie ouest a mis à nue un alignement de quelques dalles calcaires qui laisse suggérer la présence d'un muret (fig.5). 


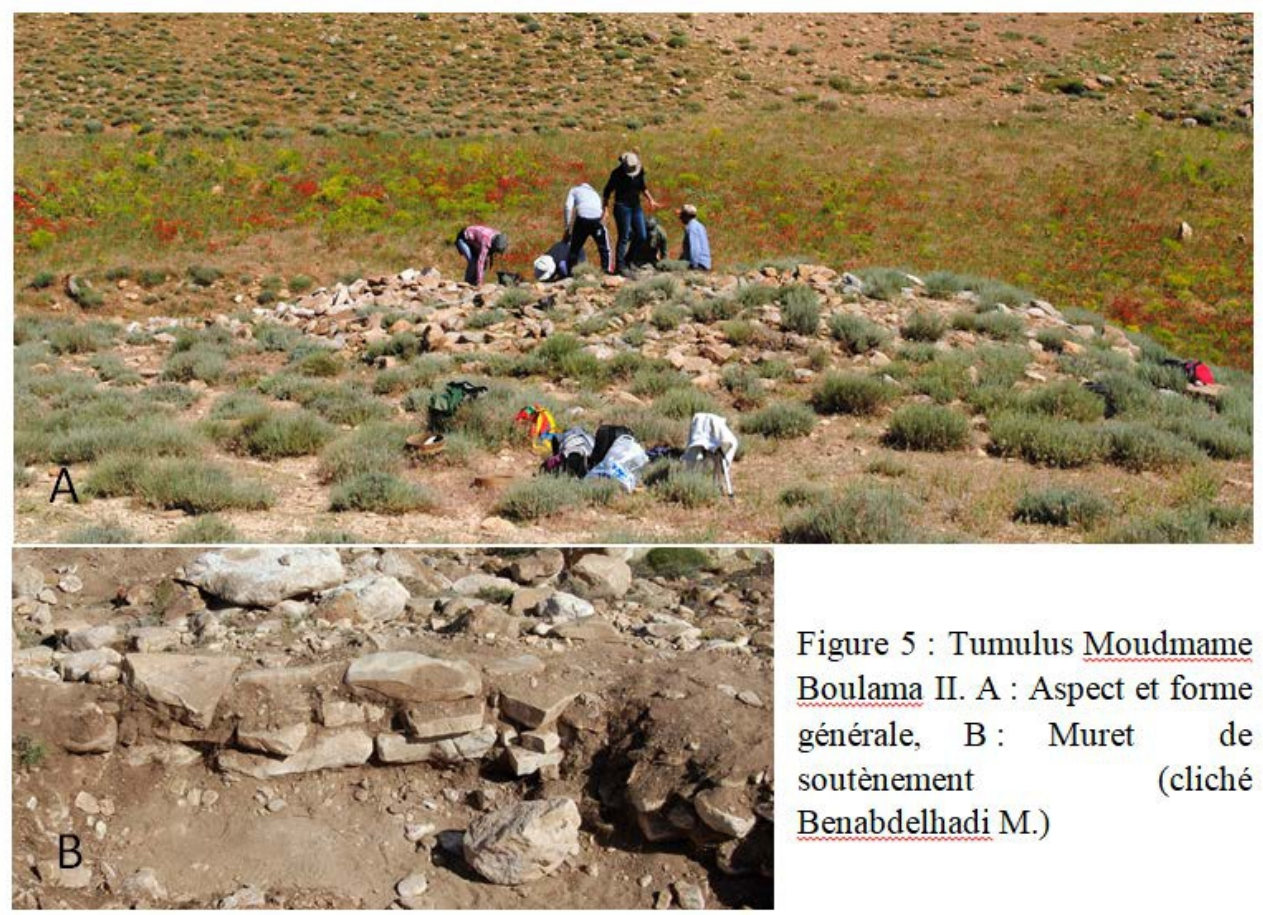

Les pierres utilisées pour la construction de ce tumulus sont en majorité en calcaire du Lias moyen, mais on trouvea également des grés du Crétacé local. Les pierres de petite de taille sont placées au sommet, alors que les grandes sont déposées sur les bordures. La taille de ces blocs peut atteindre un diamètre de 0,80 $\mathrm{m}$, une largeur de $0,67 \mathrm{~m}$ et une épaisseur de $0,50 \mathrm{~m}$.

\section{Muret de soutènement}

La partie ouest du monument présente un muret de 4,10 m de long constitué d'un aménagement de dalles placées horizontalement avec des intercalations limono-sableuses. Ces dalles en calcaires présentent des extrémités angulaires. Leur longueur varie entre 30 et $40 \mathrm{~cm}$, leur largeur est de $30 \mathrm{~cm}$ et leur épaisseur varie entre 11 et $30 \mathrm{~cm}$. La construction du muret du côté de la vallée peut expliquer sa fonction de soutènement.

\section{Niveaux architecturaux}

De point de vue architecture, le tumulus est constitué de trois niveaux qui servent à la conservation et à la condamnation des dépôts. Le premier niveau I est composé par la partie supérieure du tertre. Il est formé par une couche de pierres gréseuses et calcaires de formes irrégulières. Leur longueur varie entre 50 et $80 \mathrm{~cm}$ et leur taille tend à diminuer au sommet. Ce niveau constitue le revêtement externe du tumulus. 
Le deuxième niveau, situé à $50 \mathrm{~cm}$ en dessous de la surface du tumulus, renferme plusieurs grands blocs arrondis dont le diamètre varie entre $50 \mathrm{~cm}$ et $70 \mathrm{~cm}$. La forme et la taille de ces blocs suggèrent une origine alluviale ce qui suppose qu'ils aient été transportés à plusieurs ou à l'aide d'animaux depuis le lit de la rivière situé $10 \mathrm{~m}$ plus bas (fig.6). En se rapprochant du centre du tumulus, la forme des blocs change et devient plus plate.

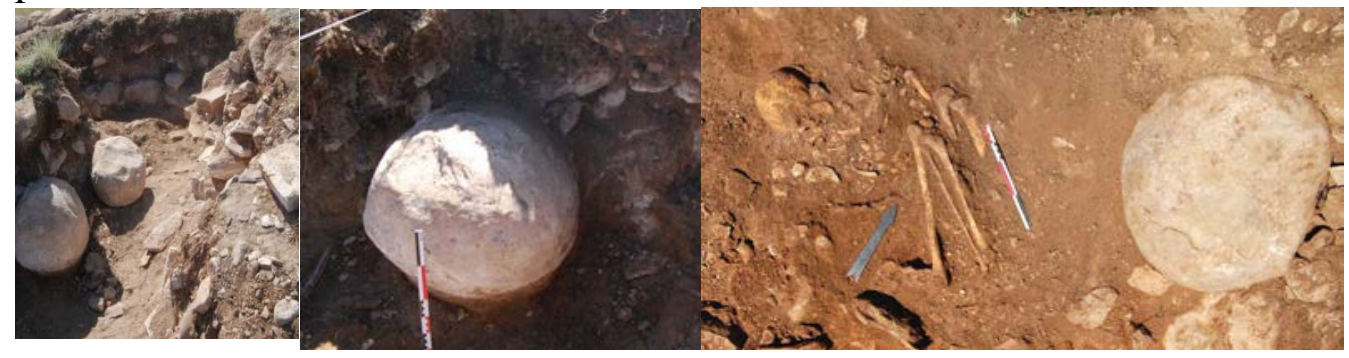

Figure 6 : Blocs arrondis du niveau II. La sépulture de H3 apparait dans le niveau III à la base de ce niveau II à blocs.

Le troisième niveau III est formé de dalles calcaires rectangulaires d'environ $80 \mathrm{~cm}$ de longueur, $45 \mathrm{~cm}$ de largeur et $15 \mathrm{~m}$ d'épaisseur qui s'intercalent avec un sédiment limono-sableux. Des restes humains et fauniques ont été mis au jour dans ce niveau (fig.7). Les ossements humains ne présentent aucune connexion anatomique et appartiennent à deux sujets ; un adulte (H1) et un immature (H2).

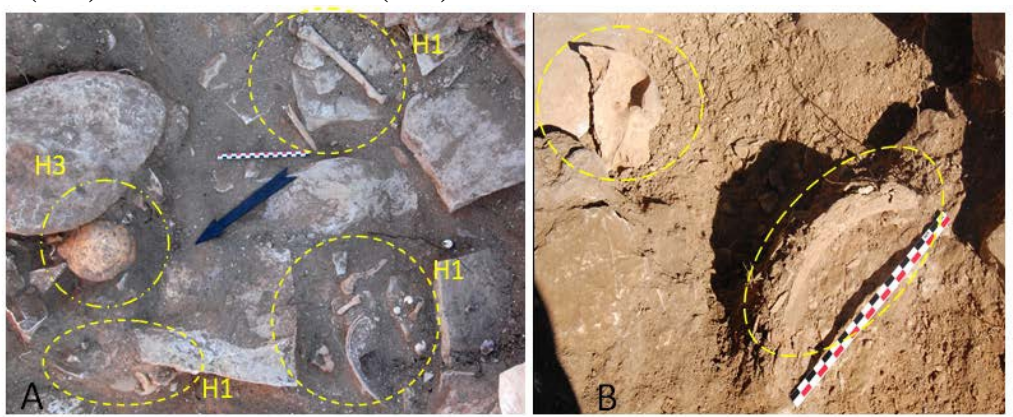

Figure 7 : Niveau III ; A : Vestiges humains (H1 et H3); B : Ossements de faune ; fragment de crâne et un bois.

\section{Fosse sépulcrale}

Le quart sud-ouest du tumulus abrite la fosse sépulcrale. Elle a une forme ovalaire et mesure environ $80 \mathrm{~cm}$ de longueur et $50 \mathrm{~cm}$ de largeur. Le fond de la fosse n'est pas horizontal ; il présente une inclinaison du côté ouest et sa structure encaissante est délimitée par un ensemble de pierres qui soutiennent ses bords. La présence d'un limon sableux de remplissage donne 
un aspect homogène à la fosse dans laquelle un seul squelette humain (H3) a été déposé.

\section{Etude archéothanatologique de la sépulture de l’individu (H3) Position des ossements}

Les ossements sont emballées dans un sédiment limono-sableux. Le squelette est celui d'un adulte masculin, inhumé en position décubitus dorsal, orienté est-ouest, la tête à l'ouest et les pieds à l'est (fig.8). Il se présente en flexion forcée et repose sur une fosse à plan ovalaire qui a été légèrement creusée par rapport au niveau original du sol et délimitée par des pierres. Les os du bassin occupent une position haute par rapport au reste du squelette, ils sont déposés contre le bord d'une paroi endurcie. Les bâtisseurs de la tombe ont probablement commencé le creusement par la partie ouest de la fosse, puis ils ont constaté la présence d'une pierre, qu'ils ont utilisée comme un bord situé du côté est. Sous l'effet de l'étroitesse de la fosse, le calvarum et la mandibule ont subi une rotation, ils sont déposés sur le côté droit. Un grand bloc a été posé sur le crâne, très probablement pour le protéger, il a une forme arrondie comme les blocs du deuxième niveau. Les membres supérieurs sont repliés et déposés sur la partie supérieure du corps : les os de l'avant-bras droit sont situés sur l'humérus droit et la main droite est placée sur l'épaule droit directement en aval du front. La main gauche, située en vue dorsale, repose sur l’épaule gauche. Les membres inférieurs sont fléchis; les deux genoux font un angle de $20^{\circ}$. Le squelette se caractérise par le maintien en connexion d'un certain nombre d'éléments labiles (les poignets des mains, la colonne cervicale et la partie distale des pieds). On notera aussi la jonction des articulations fragiles (l'articulation scapulo-thoracique). Ces arguments permettent d'affirmer qu'il s'agit d'une sépulture primaire individuelle en espace colmaté (Duday et al, 1990). La dislocation des différents étages de rachi s'est faite partiellement, donnant lieu à une colonne segmentée en sous unités composées de plusieurs ensembles de vertèbres. Cette dynamique de dislocation a provoqué différentes disjonctions produites, soit par rotation, comme c'est le cas des vertèbres cervicales qui apparaissent en position latérale gauche, soit par angulation, comme c'est le cas des quatre vertèbres lombaires qui apparaissent en vue antérieure. Par ailleurs, la cinquième lombaire a été complètement bousculée et déplacée vers le côté droit, à proximité de l'ilium du coxal droit (fig. 8). Les scapulas sont mises à plat, mais elles restent légèrement obliques en épousant le fond peu incliné de la fosse. Les clavicules ont la même position des scapulas. Cet effet est induit par la forme étroite et peu oblique de la fosse sépulcrale. Les deux coxaux sont en connexion direct avec le sacrum et ont tendance à basculer vers l'arrière en direction de l'espace libéré par les masses des muscles fessiers. 


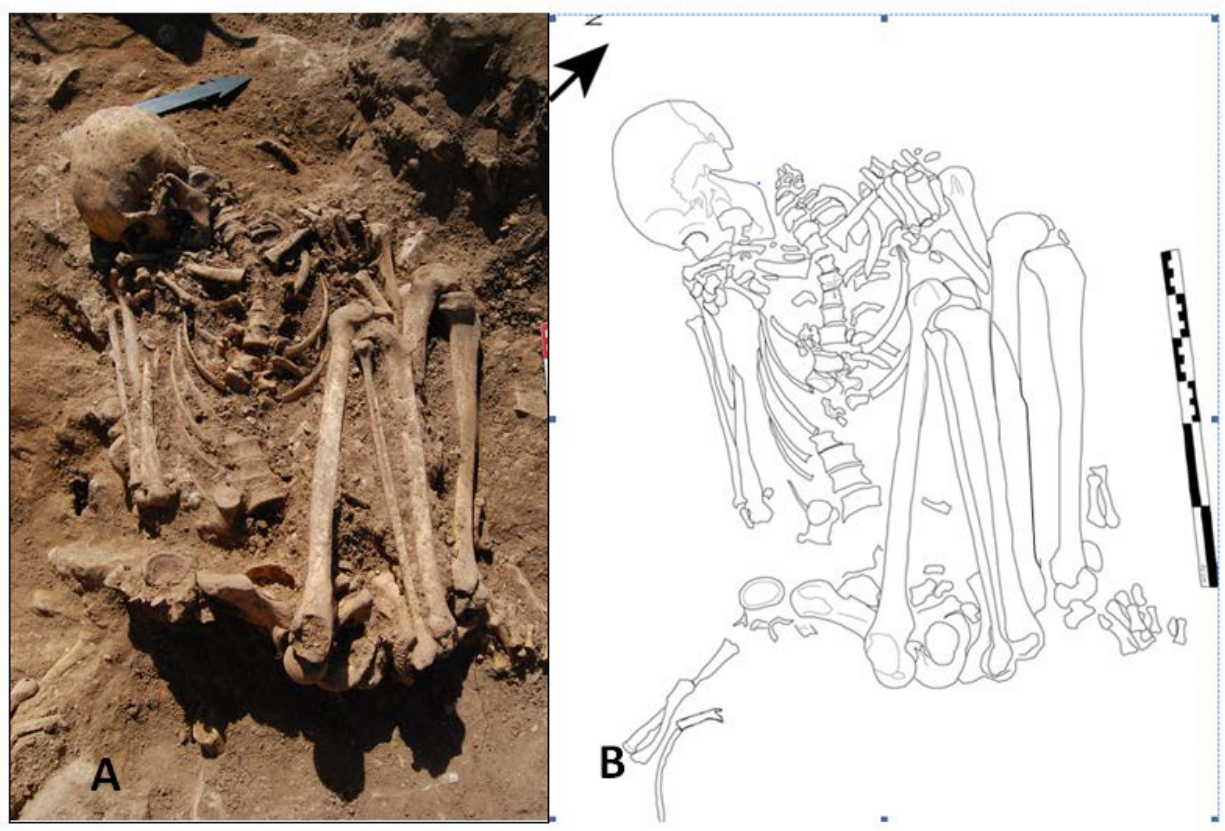

Figure 8 : A - sépulture primaire d'un individu adulte H3, de sexe masculin.

B - relevé de la sépulture de H3.

\section{Etude anthropologique}

Etats de conservation des restes osseux

Les ossements recueillis lors de la fouille présentaient généralement un très bon état de conservation. La mandibule un peu mince et déminéralisée s'est brisée lors de son nettoyage. La scapula gauche et quelques côtes présentent des fractures. Le squelette est complet et la majorité des os sont généralement en bon état de conservation. Seules quelques vertèbres (une cervicale et trois thoraciques) présentent un état de fragmentation avancé (fig. 9).

\section{Âge au décès}

L'estimation de l'âge au décès est réalisée à partir de l'examen des ossements et des molaires. Notre sujet présente des ossements de grandes dimensions avec une croissance achevée. Les épiphyses des os longs sont toutes soudées aux diaphyses et le degré de l'éruption de la troisième molaire est avancé ce qui montre que nous sommes en présence d'un sujet adulte (Thomas, 1985; White et Folken, 2005). 


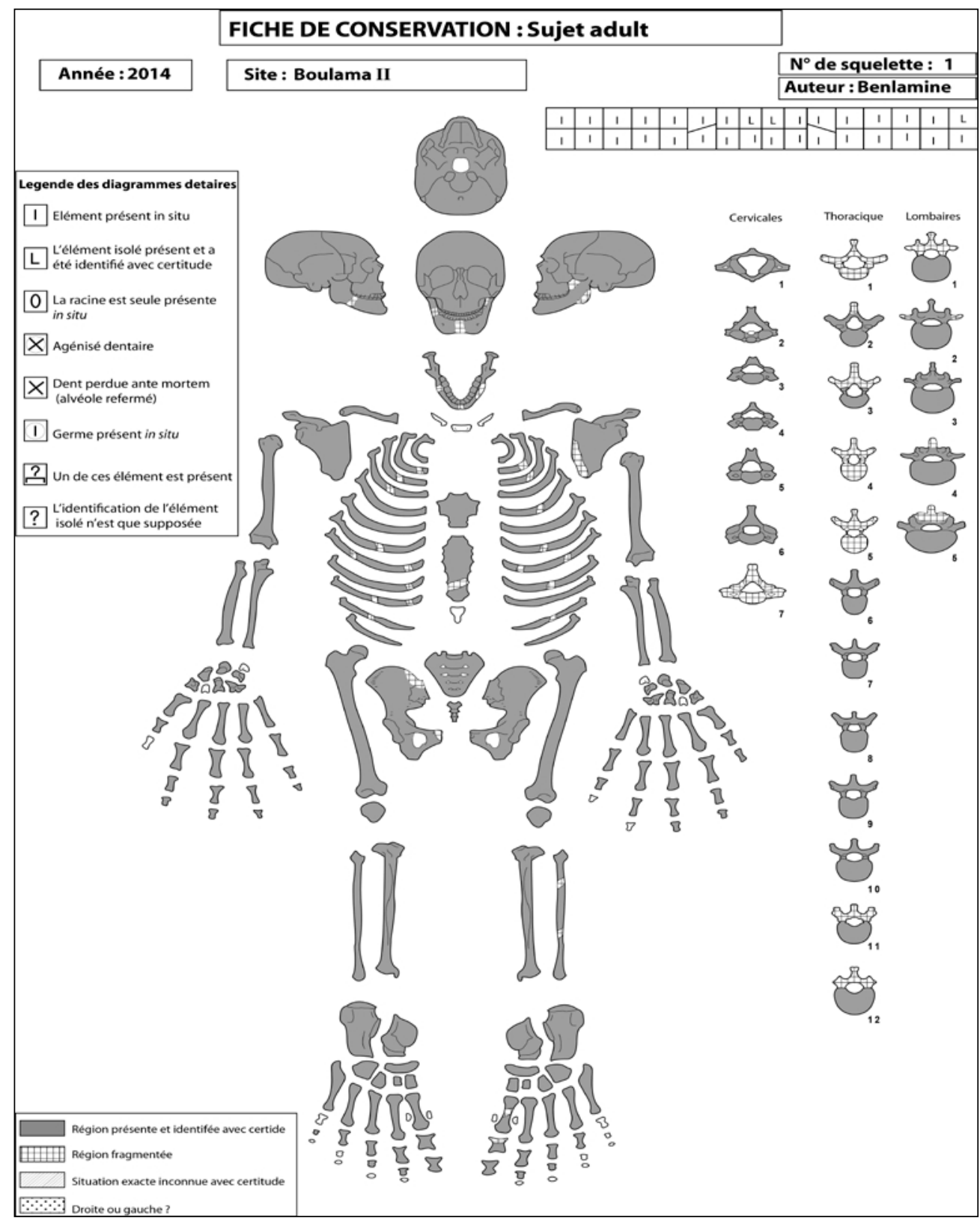

Figure 9 : Conservation du squelette de l'individu H3 selon la méthode de ConstandseeWestermann et Miklejohn.

\section{Diagnostique du sexe}

Les os du sujet $\mathrm{H} 3$ sont de grandes dimensions et présentent des reliefs d'insertion musculaire accentués. Ces caractères sont généralement masculins. Nous constatons que le bassin et la fosse iliaque sont hauts et étroits, et que la crête iliaque est contournée en ' $S$ '. La distinction sexuelle est basée d’abord sur l’os coxal puisqu'il présente une grande fiabilité, puis 
elle est confirmée par d'autres caractères morphologiques au niveau du crâne (Ferembach et $a l, 1979$ ), c'est ainsi que la hauteur et l'angle d'ouverture de la grande échancrure sciatique montre que nous sommes en présence d'un individu de sexe masculin.

\section{Détermination de la stature}

L'estimation de la stature est faite à partir des mesures des longueurs maximales d'un ou plusieurs os longs conformément aux méthodes d'Olivier et Tissier (1978). Les mesures des longueurs maximales des humérus, radius, ulnas, fémurs, tibias et les fibulas étudiés ont permis de faire des calculs fiables au vu du bon état de conservation de ces os. La stature estimée par la moyenne des valeurs calculées pour l'individu H3 est élevée puisqu'elle aurait mesuré $175 \mathrm{~cm}$.

\section{Mobilier funéraire}

La sépulture de l'individu H3 déposé dans la fosse, est dépourvue de mobilier funéraire. Quelques ossements d'une mégafaune ont été recueillis dans les sédiments du troisième niveau à coté de l'individu adulte (H2) qui présente un squelette incomplet. Il s'agit d'un bois, d'une partie du crâne et des dents isolées d'un bovidé domestique en très bon état de conservation. L'ensemble des ossements trouvé de H1, de $\mathrm{H} 2$ et ceux de la faune, se trouvent dans des endroits différents à côté de la fosse ce qui est en faveur de l'hypothèse de leur déplacement pour permettre le creusement de la fosse pour l'accueil du troisième défunt (H3). La présence de cette faune associée dans cette sépulture multiple atteste d'un rite d'offrande pour les défunts. Une datation absolue de ces restes fauniques a permis d'attribuer à ce monument funéraire un âge de $2290 \pm 35$ ans ${ }^{14}$ C B.P (Gif A15085/ Sac A 41509.

\section{Conclusion}

Le monument funéraire protohistorique MBII est un tumulus formé par une accumulation de pierres et de blocs calcaires et gréseux et caractérisé par la présence d'un muret de soutènement. L'architecture du tumulus est constituée par trois niveaux. Le niveau le plus profond (niveau III) a révélé quelques restes humains sans aucune connexion anatomique de deux individus incomplets (un adulte $\mathrm{H} 1$ et un immature $\mathrm{H} 2$ ).

La fosse sépulcrale située dans le quart sud-ouest du tumulus a livré un troisième individu (H3), inhumé en position de décubitus dorsal hyper contracté. Le squelette est complet et les ossements se présentent en connexion anatomique. C'est une sépulture individuelle primaire dans un espace colmaté. L'étude anthropologique du squelette montre qu'il appartient à un sujet adulte de sexe masculin. 
Ce monument funéraire aurait donc pu être été utilisé dans un premier temps pour l'accueil des deux individus (H1 et H2) et ensuite réutilisé pour l'inhumation de l'individu H3 dans une fosse sépulcrale. La réutilisation du monument funéraire pourrait s'expliquée par l'appartenance des trois individus à la même famille, et que le dernier sujet inhumé H3 occuperait une position familiale et probablement sociale plus importante. Cependant cette hypothèse devrait être vérifiée par des analyses de l'ADN.

La position en flexion forcée de l'individu (H3) est probablement due à l'étroitesse de la fosse qui présente une forme ovalaire un peu oblique et ne dépasse pas un mètre de longueur et $40 \mathrm{~cm}$ de largeur. C'est une position particulière et différente des autres sépultures que nous avons découvertes dans cette région du moyen Atlas. D’autre part, le substrat n’est pas dur au point d'empêcher l'élargissement de la fosse sépulcrale. Ces données nous laissent penser que le choix d'une fosse étroite et peu profonde est intentionnel.

Cette position de décubitus dorsal hyper contracté est connue chez les Ibéromaurusiens de la nécropole d'Afalou Bou Rhummel depuis le Paléolithique supérieur. Selon les descriptions d’Arambourg (1934), le niveau supérieur de ce gisement comprend six individus complets : deux sujets sont inhumés en position de décubitus latéral fléchi et quatre individus sont inhumés en position de décubitus dorsal fortement fléchi. C’est dans la culture Ibéromaurusienne que se sont développées les nécropoles en Afrique du nord. Elles présentent ces types d’inhumations intentionnelles dans un espace aménagé et selon une position déterminée du défunt.

Les rituels funéraires, comme l'aménagement du site et la position d'inhumation, sont des éléments culturels marquants lorsqu'ils sont spécifiques et reproduits tels quels sur une longue période. Ils sont transmis de génération en génération et s’enracinent ainsi dans la mémoire collective qui forme le socle de toute civilisation (Hachi, 2006). La position de décubitus dorsal hyper contracté est un rite funéraire qui trouve son origine au cours de la période de la culture ibéromaurusienne. Il s’est prolongé tout au long de la protohistoire nord-africaine et n’a été abandonné que durant la période romaine (Bokbot, 1991).

Le monument funéraire MB II est un tumulus de grande taille, situé au sommet d'une colline. Il est entouré de tumulus de petites dimensions dont il se différencie par la présence d'un muret de soutènement. On peut penser que c'est autour des sépultures d'un ou de quelques individus ayant des statuts sociaux particuliers, que viennent s'agréger les tombes de personnes plus anonymes et qu'ainsi se forment les nécropoles. L’inhumation intentionnelle possède généralement des aménagements particuliers qui permettent de reconnaître le statut social du défunt (Hachi, 2006). L'architecture particulière du tumulus et l'inhumation du squelette 
en position de décubitus dorsal hyper-contracté, sont considérés comme des aménagements intentionnels bien soignés. Autrement dit, L'individu H 3 a été inhumé avec soin dans un lieu choisi et bien aménagés. Ces données permettent de postuler que cet individu présentait un statut social particulier.

\section{References:}

1. Arambourg C. M. Boule, H. Vallois, R. Verneau (1934) : Les grottes paléolithiques des Beni-Ségoual (Algérie), Archives de l'IPH, mémoire $\mathrm{n}^{\circ} 13,252 \mathrm{p}$.

2. Benabdelhadi M., Ouja A., Zouhir S., Ouchaou B., Boudad L., Fontune M., Simonic C., Amans A. Gourari L. (2007) : Recherches sur la Préhistoire et la Géologie du Quaternaire pour le développement socioculturel de la région du Moyen Atlas, Rapport de fouille, Fès, Faculté des Sciences et Technique, Fès Sais, 22 p.

3. Benabdelhadi M., Oujaa A., Charroud M., Ouchaou B., Zouhri S., Boudad L., Binou K., Simonis C., Puccini D., Fontugne M. (2008) : Nouveaux sites préhistoriques dans le Moyen Atlas marocain: résultats des premières recherches. Le Quaternaire marocain dans son contexte méditerranéen, Acte de la Quatrième Rencontre des Quaternaristes Marocains ; RQM4, Vol 2, Oujda, 2008, Ed. Faculté des sciences Oujda, p. 265-279.

4. Bokbot Y. (1991): Habitats et monuments funéraires protohistoriques du Maroc protohistorique, Thèse de doctorat, Université de Provence Aix-Marseille I, Marseille, 549 p.

5. Constandsee-Westermann, T.-S. et Meiklejohn, C. (1978) : The humain skeletal material from Swifterbant, earlier Neolithic of the Northern Netherlands. I. Inventory and demography,

6. Palaeohistoria, XX : pp. 39-89.

7. Duday, H., Courtaud, P., Crubezy, E., Sellier, P. et Tillier, A.-M., (1990); L'Anthropologie de terrain : Reconnaissance et interprétation des gestes funéraires. Bull. et Mém. de la Soc. d'Anthrop. de Paris, n. s., t. 2, n 3-4, p 39.

8. Ferembach, D., Schwidetzky, I. et Stloukal, M., (1979) : Recommandations pour déterminer l'âge et le sexe sur le squelette. Bull. Mém. Soc. d'Anthrop. de Paris, 6, XIII, pp. 9-10.

9. Hachi, S. (2006): Du comportement symbolique des derniers chasseurs Mechta-Afalou d'Afrique du Nord, Comtes Rendus, Pale. vol 5, Elsevier Ed, p 429 - 440.

10. Olivier G et Tissier H. (1978) : Détermination de la stature et de la capacité crânienne, Bull. Mém. Soc. Anthropologie de Paris, Tome 2, Série XIII, p.297-306. 
11. Thomas A. (1985) : Elément de Paléoanthropologie, Publication de l'Institut Supérieur d'Archéologie et d'Histoire de l'Art, Louvain La neuve, 18, p. 60-90.

12. Wites T. et Folken P. (2005) : The Human Bone Manual, Academic Press, Elsevier Ed, 464 p, 2005. 